\title{
The Role of Density and Temperature in the Dynamics of Polymer Blends
}

\author{
C. M. Roland ${ }^{*, \dagger}$ and R. Casalini ${ }^{\dagger}, \star$ \\ Chemistry Division, Code 6120, Naval Research Laboratory, Washington, D.C. 20375-5342, and \\ Chemistry Department, George Mason University, Fairfax, Virginia 22030
}

Received July 7, 2005; Revised Manuscript Received August 17, 2005

\begin{abstract}
The relative effect of volume and thermal energy on the local segmental dynamics, as reflected in the ratio of the isochoric, $E_{V}$, and isobaric, $E_{P}$, activation enthalpies, is determined for blends of polystyrene (PS) with poly(2,6-dimethyl-1,4-phenylene oxide) (PPO) and with poly(vinyl methyl ether) (PVME). We find that neat PPO near $T_{\mathrm{g}}$ has the lowest value of $E_{V} / E_{P}=0.25 \pm 0.02$ reported for any polymer, indicating volume-dominated dynamics. Addition of the lower $T_{\mathrm{g}} \mathrm{PS}$ alleviates constraints on local motion, resulting in a weaker volume effect. The opposite situation prevails with PS/PVME blends. PS has a higher $T_{\mathrm{g}}$, and in blends segmental relaxation of the PVME becomes more controlled by volume than for neat PVME. We also show herein that the relaxation times for the PVME/PS blends measured at various $T$ and $P$ superpose when plotted vs $T v^{\gamma}$, where $v$ is the specific volume and $\gamma$ is a material constant. This scaling, which has previously been demonstrated for various neat glass-formers and is reported herein for the first time for a blend, enables $E_{V} / E_{P}$ to be determined at $T_{\mathrm{g}}$ in the absence of actual measurements near $T_{\mathrm{g}}$.
\end{abstract}

\section{Introduction}

Although polymer blends are of obvious practical utility (finding applications as diverse as those of neat polymers $\left.{ }^{1-5}\right)$, fundamental studies of blends and mixtures can also be productive, with phenomena observed having no counterpart in pure materials. These new effects can offer stern tests of theoretical models. Much recent research on blends has focused on their relaxation behavior, both the local segmental dynamics and the global chain motions. While the latter are unique to macromolecules, segmental relaxation of polymers is of more general interest, since it underlies the global dynamics and has many properties in common with structural relaxation of simple, molecular liquids.

There are a number of extant models for blend dynamics, ${ }^{6-12}$ each accounting in somewhat different fashion for the salient features: thermorheological complexity, low-frequency broadening of the dispersion in the dielectric or mechanical loss spectra, dynamic heterogeneity (i.e., distinct relaxation peaks for each component), etc. Other phenomena, which may be specific to certain blends or particular measurement conditions, are not so easily described by available models; these include relaxation times that are not intermediate between those of the pure components, ${ }^{13-16}$ the composition independence of the relaxation times seen at very high temperatures, ${ }^{17,18}$ and the effect of blending on secondary relaxations. ${ }^{19}$

Accounting for the dynamics in blends obviously requires an understanding of the relaxation behavior of the neat components. Recent progress along these lines has come from the use of pressure as an experimental variable. By measuring relaxation times as a function of $T$ and $P$ (and thus also of volume), the relative degree to which thermal energy and density govern the dynamics can be quantified. ${ }^{20-24}$ The usual approach is to determine the ratio of the activation

\footnotetext{
Naval Research Laboratory.

$\$$ George Mason University.
}

enthalpies at constant volume, $E_{V}=\left.R\left(\mathrm{~d} \ln \tau_{\alpha} / \mathrm{d} T^{-1}\right)\right|_{V}$, to that at constant pressure, $E_{P}=\left.R\left(\mathrm{~d} \ln \tau_{\alpha} / \mathrm{d} T^{-1}\right)\right|_{P}$, where $\tau_{\alpha}$ is the relaxation time for the glass transition. $E_{V} / E_{P}$ can vary from 0 (corresponding to volume dominated dynamics) to unity (temperature as the control variable) and for polymers has been found to fall in the range from 0.5 to 0.8 at temperatures near $T_{\mathrm{g} .}{ }^{24,25}$ This means that the change in local segmental relaxation time upon cooling toward the glass temperature is due to both thermal contraction of the material and its energy loss, with the latter exerting a somewhat stronger effect $\left(E_{\mathrm{V}} / E_{P}>0.5\right)$.

A recent development is our finding that $\tau_{\alpha}$, measured for various conditions of $T$ and $P$, can be superposed by plotting vs the product of the temperature times the specific volume, $v$, raised to a constant; i.e., $\tau_{\alpha}=f\left(T v^{\gamma}\right)$, where $\gamma$ varies among materials but is independent of $T$ and $P .^{26-28}$ The underlying idea is that segmental motions are thermally activated but impeded by steric constraints (jamming); this confers a density dependence to the activation energy, whereby non-Arrhenius behavior $\left(\log \tau_{\alpha}\right.$ not proportional to $T^{-1}$ ) is observed. The magnitude of the scaling exponent $\gamma$ reflects the role of volume in controlling the change in $\tau_{\alpha}$ with temperature, and in principle it may be related to the intermolecular repulsive interactions. ${ }^{26}$ If $\tau_{\alpha}$ is a function of $T v^{\gamma}$, it follows that ${ }^{26,27}$

$$
E_{V} / E_{P}=\left(1+\gamma \alpha_{P} T\right)^{-1}
$$

where $\alpha_{P}$ is the isobaric thermal expansion coefficient. Note that the product $\alpha_{P} T$ is roughly constant at $T_{\mathrm{g}}$ equal to $\sim 0.2^{27,29}$ (although an evaluation of literature data suggests a weak increase with $T_{\mathrm{g}}$ ). While $E_{V} / E_{P}$ is usually determined near $T_{\mathrm{g}}$, it varies with temperature. Equation 1 can be used to calculate $E_{V} / E_{P}$ at any temperature since $\gamma$ is a constant. The $\gamma$-scaling has recently been extended to the normal mode of polymers, ${ }^{30-32}$ with the same value of the exponent yielding superposition of both the normal mode relax- 
ation time and $\tau_{\alpha}$. This implies that both the global and local segmental relaxations are governed to the same relative degree by $T$ and $V$, although their behavior is described by different functions of the variable $T V^{\gamma}$.

In this paper we analyze relaxation and equation-ofstate data for two blends: (i) poly(2,6-dimethyl-1,4phenylene oxide) (PPO) with atactic polystyrene (PS) and (ii) poly(vinyl methyl ether) (PVME) with PS. These blends are essentially van der Waals mixtures, having only weak interactions between the components. ${ }^{33,34} \mathrm{An}$ important difference is that in the first case PS is added to a polymer (PPO) having a higher $T_{\mathrm{g}}$, while in the second case PS is mixed with a lower $T_{\mathrm{g}}$ material (PVME). Our principal interest concerns the influence that volume and temperature have on segmental relaxation in the blends, in comparison to the neat components. Current models of blend dynamics restrict their consideration to the variation of $\tau_{\alpha}$ with $T$, and only a few experimental studies have addressed the effect of pressure on $\tau_{\alpha}$ for mixtures, ${ }^{28,35,36}$ block copolymers,${ }^{37}$ or blends. ${ }^{38-41}$ Fundamental understanding, however, requires analysis of what governs the observed dependences. At least for the two blends studied herein, we find that the effect of added PS on $E_{V} / E_{P}$ depends on the relative $T_{\mathrm{g}}$ of the components. $T_{\mathrm{g}}$ of PS is intermediate between that of PPO (largest) and PVME and increases $E_{V} / E_{P}$ for the former while decreasing it for the latter blend. Thus, the relative relaxation times of the components, as reflected in their neat $T_{\mathrm{g}}$, appear to govern how blending influences the effect of volume on $\tau_{\alpha}(T)$. We also examine whether the $\log \left(\tau_{\alpha}\right)=f\left(T v^{\gamma}\right)$ scaling of neat materials also applies to PVME in blends with PS.

\section{Results}

PPO/PS Blends. One of the early commercially significant polymer blends is PPO and PS (General Electric's Noryl). A limited number of studies of the dynamics in this blend have been reported. Optical and infrared birefringence measurements were used to follow chain orientation in stretched films. ${ }^{42-44}$ Chain relaxation of the components was found to be "coupled" due to the homogeneous phase morphology. ${ }^{42}$ Timetemperature shift factors for the chain dynamics are only weakly dependent on composition..$^{45}$ NMR has been used to study the component dynamics near the glass transition ${ }^{46,47}$ and the effect of blending on motion of the phenyl side groups in PPO/PS blends. ${ }^{48}$ Concerning the local segmental relaxation, Robertson and Wilkes ${ }^{49}$ reported an increased blend fragility $\left(T_{\mathrm{g}}\right.$-normalized temperature dependence of $\tau_{\alpha}$ ) relative to the neat components.

$P V T$ measurements have been carried out on neat PPO and various compositions with PS, 50,51 and in Figure 1 we show representative results for the specific volume of neat PPO as a function of temperature at pressures from ambient to $120 \mathrm{MPa}$. For the respective liquid and glassy states (but well away from the glass transition), $v$ can be fit to quadratic functions whose intersection defines $T_{\mathrm{g}}$. These $T_{\mathrm{g}}$ values are indicated in Figure 1 and yield the pressure coefficient of the glass transition. Results for the PPO and three blends with PS are given in Table 1 . For neat PPO, $\mathrm{d} T_{\mathrm{g}} / \mathrm{d} P=804$ $\mathrm{K} / \mathrm{GPa}$, which is the largest pressure coefficient of $T_{\mathrm{g}}$ ever reported for any molecular liquid or polymer. ${ }^{25}$

In Figure 2 the ambient pressure $T_{\mathrm{g}}$ is plotted vs composition, together with the value for neat PS (also

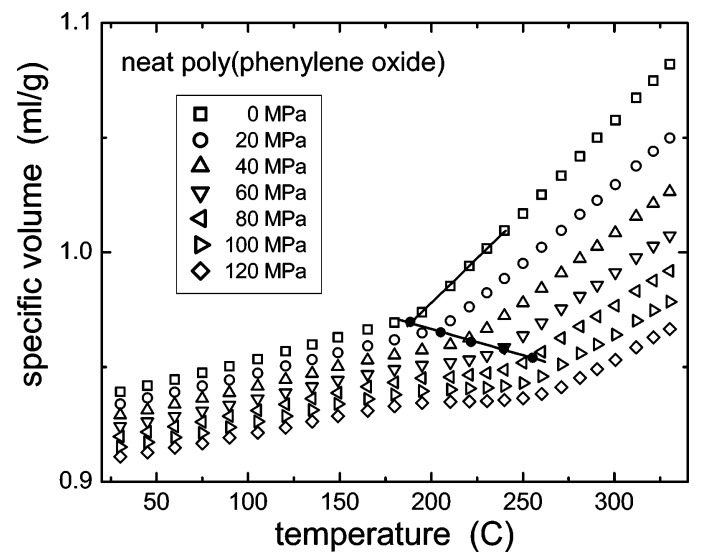

Figure 1. Specific volume vs temperature for neat PPO at the indicated pressures (data from ref 51). The solid circles represent $T_{\mathrm{g}}(P)$ as determined from the intersection of the liquid and glassy $v(T)$ data. Solid lines are the isobaric $(P=$ 0 ) and isochronal $\left(\tau\left(T_{\mathrm{g}}\right)\right)$ fits.

Table 1. Results for PPO/PS Blends

\begin{tabular}{cccc}
\hline PPO $(\%)$ & $T_{\mathrm{g}}\left({ }^{\circ} \mathrm{C}\right)$ & $\begin{array}{c}\lim _{P \rightarrow 0}\left(\mathrm{~d} T_{\mathrm{g}} / \mathrm{d} P\right) \\
(\mathrm{K} / \mathrm{GPa})\end{array}$ & $E_{V} / E_{P}\left(\right.$ at $\left.T_{\mathrm{g}}\right)$ \\
\hline 100 & 188.6 & $840 \pm 8$ & $0.25 \pm 0.02$ \\
70 & 153.3 & $692 \pm 15$ & $0.35 \pm 0.02$ \\
50 & 124.6 & $555 \pm 23$ & $0.50 \pm 0.04$ \\
30 & 112.8 & $483 \pm 20$ & $0.51 \pm 0.03$ \\
0 & $100^{a}$ & $360 \pm 50^{a}$ & $0.64 \pm 0.05^{a}$
\end{tabular}

${ }^{a}$ Reference 52 .

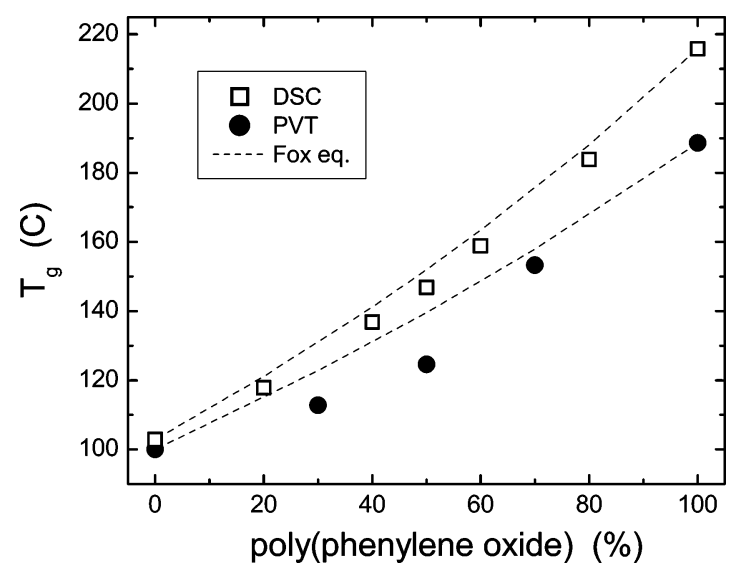

Figure 2. Composition dependence of the glass transition temperature of PPO/PS blends, determined herein from $P V T$ data and as reported in ref 55 from calorimetry. The dotted lines are the respective fits to eq 2 .

obtained from $P V T$ data $^{52}$ ). It is common in models of blend dynamics ${ }^{8,11}$ to employ the Fox equation to describe the composition dependence of $T_{\mathrm{g}}{ }^{53}$

$$
T_{\mathrm{g}}\left(\phi_{1}\right)=\left(\frac{\phi_{1}}{T_{\mathrm{g}, 1}}+\frac{1-\phi_{1}}{T_{\mathrm{g}, 2}}\right)^{-1}
$$

The fact that the Fox equation is readily implemented (no adjustable parameters) accounts for much of its popularity. Its limitations have led to modifications entailing additional parameters. ${ }^{54}$ As seen in Figure 2, eq 2 overestimates $T_{\mathrm{g}}$ for the blends by $\leq 10 \%$. Also included in the figure are DSC results ${ }^{55}$ for a PPO/PS blend of comparable molecular weights. There is a similar negative deviation from eq 2 .

The glass transition temperature determined from $P V T$ measurements corresponds to the temperature at 


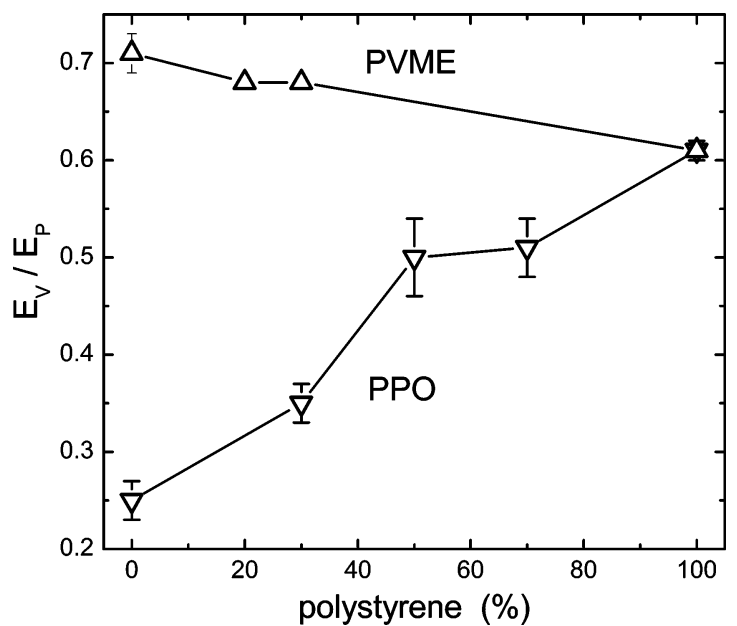

Figure 3. Activation enthalpy ratio as a function of composition for PVME/PS $(\triangle)$ and PPO/PS $(\nabla)$ blends. An increasing value of $E_{V} / E_{P}$ denotes stronger effect of thermal energy, relative to that of volume, on $\tau_{\alpha}(T)$.

which $\tau_{\alpha}$ is constant; that is, $\tau_{\alpha}\left(T_{\mathrm{g}}\right)$ is pressureindependent. This means that while $\tau_{\alpha}\left(T_{\mathrm{g}}\right)$ may vary for different materials (usually being in the vicinity of 100 s) and the value of $T_{\mathrm{g}}$ itself is rate dependent, for a given material whose volume is measured at fixed rate the relaxation time at $T_{\mathrm{g}}$ does not change with pressure. ${ }^{25}$ This means that the specific volume at $T_{\mathrm{g}}(P)$ can be used to define an isochronic thermal expansion coefficient, $\alpha_{\tau}$ $\left(\left.\equiv v^{-1}(\mathrm{~d} v / \mathrm{d} T)\right|_{\tau}\right)$. Ferrer et al. ${ }^{22}$ have shown that the ratio of $\alpha_{\tau}$ and the isobaric thermal expansion coefficient, $\alpha_{P}$, provides another measure of the relative degree to which volume and temperature govern $\tau_{\alpha}(T)$. The activation enthalpy ratio is related to these thermal expansivities according to ${ }^{56}$

$$
E_{V} / E_{P}=\left(1-\alpha_{P} / \alpha_{\tau}\right)^{-1}
$$

Using eq 3 , together with the assumption that $\tau_{\alpha}\left(T_{\mathrm{g}}\right)$ is constant, allows $E_{V} / E_{P}$ to be calculated without measurement of relaxation times. The isobaric thermal expansion coefficient above $T_{\mathrm{g}}$ is determined directly from the PVT data, while $\alpha_{\tau}=v\left(T_{\mathrm{g}}\right)^{-1}\left[\mathrm{~d} v\left(T_{\mathrm{g}}\right) / \mathrm{d} T_{\mathrm{g}}(P)\right]$. The $E_{V} / E_{P}$ so obtained for PPO as a function of composition are plotted in Figure 3, along with the value for neat PS. ${ }^{52}$ The uncertainty reflects scatter in the $T_{\mathrm{g}}(P)$ data.

For neat PPO, the enthalpy ratio is quite small, $=0.25$, the lowest found to date for any polymer ${ }^{25}$ This indicates that the temperature dependence of the segmental dynamics of PPO is governed primarily by changes in volume, with thermal energy exerting a relatively small effect. The low value of $E_{V} / E_{P}$ for PPO follows from the magnitude of its pressure coefficient of $T_{\mathrm{g}}$, since this contributes to a large (negative) $\alpha_{\tau}$. Addition of the lower $T_{\mathrm{g}}$ PS increases $E_{V} / E_{P}$; that is, the presence of PS makes $\tau_{\alpha}$ less dependent on density. Interestingly, blending causes densification of the blendas shown in Figure 4 , the specific volume at $T_{\mathrm{g}}$ passes through a minimum vs composition. This is normal behavior for mixtures with strong specific interactions and can also arise from local packing effects. ${ }^{57,58}$

PVME/PS Blends. This is one of the most studied polymer blends, due to the convenient $T_{\mathrm{g}}$ and its miscibility despite weak interactions. Dielectric spectroscopy measurements on PVME/PS ${ }^{39,59-61}$ are especially interesting since the dynamics of the polar PVME

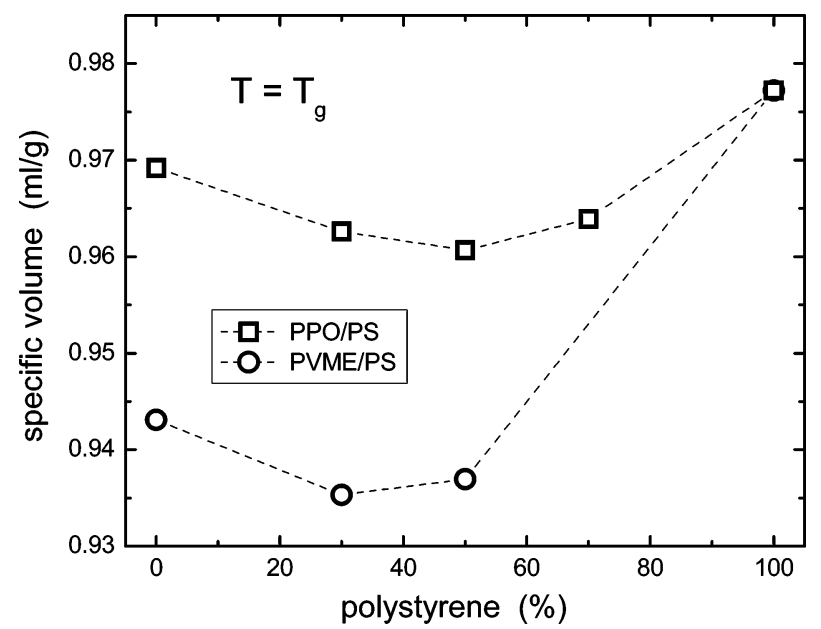

Figure 4. Composition dependence of $v$ for PVME/PS and PPO/PS blends. Both have a negative excess volume.

are monitored without interference from the relatively nonpolar PS. We have previously shown ${ }^{27}$ that relaxation times for neat PVME, measured vs $T$ at ambient $P$ and at three temperatures for pressures up to 726 $\mathrm{MPa},{ }^{56}$ superpose when expressed as a function of $T v^{2.55}$. Scaling of the data of Floudas, who measured neat PVME as a function of pressure at various temperatures, ${ }^{39}$ gives an equivalent value of $\gamma$. Since for PVME and its blends with PS considered herein, $T_{\mathrm{g}}$ is below the range of the $P V T$ measurements, $E_{V} / E_{P}$ cannot be extracted directly from the $P V T$ data. Accordingly, we make use of eq 1 , relating the activation enthalpy ratio to the scaling exponent $\gamma$. From $P V T$ data for PVME in the equilibrium liquid state, ${ }^{62} \alpha_{\mathrm{P}}=5.697 \times 10^{-4} \mathrm{~K}^{-1}$ at $T_{\mathrm{g}}=247.6 \mathrm{~K}$; thus, eq 1 yields $E_{V} / E_{P}=0.73$, consistent with the value determined directly from slope of the isochoric and isobaric relaxation times vs reciprocal temperature, $E_{V} / E_{P}=0.69 .{ }^{56} \mathrm{~A}$ value this large is typical of polymers, whose segmental relaxation times are usually influenced more strongly by thermal energy than by density.

For blends of PVME with 30 and 50 wt $\%$ of PS, Floudas ${ }^{39}$ measured the dielectric relaxation times at ambient pressure and also determined the activation volumes, $\Delta V=\left.R T\left(\mathrm{~d} \log \tau_{\alpha} / \mathrm{d} P\right)\right|_{T}$, from measurements at pressures up to $200 \mathrm{MPa}$. From these data we obtain $\tau_{\alpha}(T, P)$. To express the relaxation times as a function of $T v^{\gamma}$ requires the equation of state for the blends. Using published PVT data ${ }^{50}$ for blends of PVME and PS having the same composition and (high) molecular weights similar to the samples measured dielectrically, we fit the Tait equation ${ }^{63}$

$$
\begin{array}{r}
v(T, P)=\left(a_{0}+a_{1} T+a_{2} T^{2}\right)[1-0.0894 \ln (1+ \\
\left.\left.P / b_{0} \exp \left[-b_{1} T\right]\right)\right]
\end{array}
$$

with the results given in Table 2 .

We then calculate $v$ for each $T$ and $P$ at which $\tau_{\alpha}$ is known, obtaining the master curves of the $\log \tau_{\alpha}\left(T v^{\gamma}\right)$ shown in Figure 5. Good superpositioning is achieved with $\gamma=3.0 \pm 0.05$ independent of blend composition. This is the first instance of the scaling $\log \left(\tau_{\alpha}\right)=f\left(T v^{\gamma}\right)$ shown to be valid for a blend. The scaling exponent is significantly larger than the value of 2.55 for neat PVME. From eq $1 E_{V} / E_{P}=0.68 \pm 0.01$ for the blends; these data are included in Figure 3. There is a systematic increase in the degree to which volume governs the segmental dynamics with addition of PS to the PVME. 
Table 2. Equation-of-State Parameters for PVME/PS Blends

\begin{tabular}{cccccc}
\hline PVME $(\%)$ & $v_{0}(\mathrm{~mL} / \mathrm{g})$ & $v_{1}\left(\mathrm{~mL} /\left(\mathrm{g}{ }^{\circ} \mathrm{C}\right)\right)$ & $v_{2}\left(\mathrm{~mL} /\left(\mathrm{g}{ }^{\circ} \mathrm{C}^{2}\right)\right)$ & $b_{0}(\mathrm{MPa})$ & $b_{1}\left({ }^{\circ} \mathrm{C}^{-1}\right)$ \\
\hline 50 & $0.9415 \pm 0.0002$ & $(5.30 \pm 0.04) \times 10^{-4}$ & $(4.3 \pm 0.2) \times 10^{-7}$ & $245 \pm 1$ & $(4.60 \pm 0.04) \times 10^{-3}$ \\
70 & $0.9442 \pm 0.0001$ & $(5.90 \pm 0.02) \times 10^{-4}$ & $(3.75 \pm 0.09) \times 10^{-7}$ & $225.0 \pm 0.6$ & $(4.52 \pm 0.02) \times 10^{-3}$
\end{tabular}

Figure 4 shows the specific volume at $T_{\mathrm{g}}$ for the two PVME/PS blends and the neat components. Similar to $\mathrm{PPO} / \mathrm{PS}$, there is substantial densification; that is, the excess volume is negative.

\section{Discussion and Summary}

Neat PPO has an unusually low value of $E_{V} / E_{P}=0.25$ \pm 0.02 , revealing the relative dominance of volume, as opposed to thermal energy, in determining the variation of $\tau_{\alpha}$ with $T$. For other polymers, $0.5>E_{V} / E_{P}>0.8$; that is, temperature tends to be the stronger control variable. ${ }^{24,25}$ The latter is a consequence in polymers of a plethora of intramolecular bonds, which are insensitive to pressure. This insensitivity is evidenced by the invariance to pressure of the normal mode dielectric strength, which depends on the chain end-to-end distance. ${ }^{31}$ The unusually strong effect of $v$ on the dynamics of PPO is related at least in part to the flexibility of its backbone due to the ether linkage. Volume effects are generally emphasized in flexible polymers: polysiloxanes have activation enthalpy ratios close to $0.5^{24,25}$ (equal influence of $v$ and $T$ ), and for poly(propylene oxide) $E_{V} / E_{P}=0.55$ (albeit measured for $T>T_{\mathrm{g}}$ ). ${ }^{64}$ The high $T_{\mathrm{g}}$ of PPO may also contribute to a low value of $E_{V} / E_{P}$ since very generally this ratio decreases with increasing temperature. This can be seen from inspection of eq 1 , noting that the product $\alpha_{P} T$ is an increasing function of temperature while $\gamma$ is constant. It is noteworthy that most flexible chain polymers (e.g., poly(ethylene oxide), poly(propylene oxide), siloxane polymers) are low $-T_{\mathrm{g}}$ elastomers. It may be the unique circumstance in PPO of chain flexibility yet a high $T_{\mathrm{g}}$ that gives rise to the unusually strong influence of volume on the dynamics of this polymer.

The dynamics of PPO/PS become less volume dependent with increasing concentration of PS. We expect environments enriched in PS to facilitate relaxation of PPO segments because the PS has a lower $T_{\mathrm{g}}$. This means that PS segments will tend to have relaxed when segmental relaxation of the PPO is considered. In the limit of $\tau_{\alpha}(\mathrm{PPO}) \gg \tau_{\alpha}(\mathrm{PS})$, the PPO does not participate in the cooperative dynamics of the PS and thus would

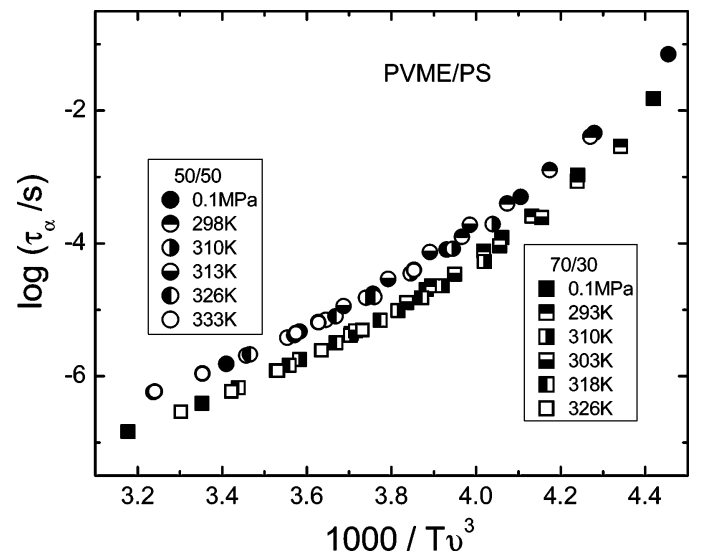

Figure 5. Specific-volume-scaled Arrhenius plots of dielectric $\alpha$-relaxation times for PVME mixed with $30 \%$ (squares) and $50 \%$ (circles) PS. The scaling exponent, $\gamma=3$, is significantly larger than the value of 2.55 for neat PVME. The experimental data are from ref 39. be unconstrained. This is a general property of blends whose components differ significantly in $T_{\mathrm{g}}{ }^{19,65}$ and is exemplified by probe molecules whose dynamics are strongly correlated with the magnitude of their relaxation time relative to that of the matrix. ${ }^{66}$ As seen herein, the mitigation of intermolecular constraints with increasing PS content leads to a decreasing effect of volume on $\tau_{\alpha}(T)$.

Opposite to the case of PPO, the dynamics of PVME become more volume dependent with increasing concentration of PS. The $T_{\mathrm{g}}$ of PS is higher than $T_{\mathrm{g}}$ of PVME, so that addition of PS constrains the dynamics of PVME, since PS is unrelaxed on the time scale of the PVME segmental relaxation. This means a PS-rich local environment is unaccommodating, and constraints on the local motion of a PVME segment are not easily mitigated. The result is increased fragility of PVME in blends with increasing PS concentration ${ }^{7}$ and, as seen herein, a stronger effect of volume on $\tau_{\alpha}$ of PVME.

The idea that the relative mobility of the local environment influences the dynamics underlies the concept of "dynamic facilitation" of Garrahan and Chandler. ${ }^{67,68}$ In their model, jammed particles (atoms or molecules) become unjammed (i.e., constraints on their local motion are alleviated) when the particles are adjacent to a region that is already unjammed. The dynamic facilitation arising in neat materials due to dynamic heterogeneity will be more prominent in blends because of the effect of concentration fluctuations. We also note that the relative effect of volume on the blend dynamics does not depend on the excess mixing volume. Blends of both PPO/PS and PVME/PS are more dense than the weighted average of their component densities, yet the effect of blending on $E_{V} / E_{P}$ is opposite for the two systems.

We find that the PVME segmental relaxation times in blends with PS conform to the $\log \left(\tau_{\alpha}\right)=f\left(T v^{\gamma}\right)$ scaling previously discovered for neat materials. This scaling is especially useful herein since $P V T$ data for $T \leq T_{\mathrm{g}}$ could not be measured for PVME/PS blends due to their low (subambient) $T_{\mathrm{g}}$. Since the scaling exponent $\gamma$ is constant, eq 1 allows $E_{V} / E_{P}$ to be determined for any temperature and pressure in the equilibrium $\left(>T_{\mathrm{g}}\right)$ state.

Acknowledgment. This work was supported by the Office of Naval Research. We thank George Floudas for providing his published data electronically.

\section{References and Notes}

(1) Plate, N. A.; Litmanovich, A. D.; Kudryavtsev, Y. V. J. Polym. Sci., Ser. A 2004, 46, 1108.

(2) Mucha, M. Prof. Polym. Sci. 2003, 28, 837.

(3) De Paoli, M. A.; Gazotti, W. A. Macromol. Symp. 2002, 189, 83.

(4) Antony, P.; De, S. K.; van Duin, M. Rubber Chem. Technol. 2001, 74, 376.

(5) Roland, C. M. In Handbook of Elastomers, 2nd ed.; Bhowmick, A. K., Stephens, H. L., Eds.; Marcel Dekker: New York, 2001; Chapter 8.

(6) Roland, C. M.; Ngai, K. L. Macromolecules 1991, 24, 2261.

(7) Roland, C. M.; Ngai, K. L. Macromolecules 1992, 25, 363; 2000, 33, 3184 .

(8) Katana, G.; Fischer, E. W.; Hack, Th.; Abetz, V.; Kremer, F. Macromolecules 1995, 28, 2714. 
(9) Kumar, S. K.; Colby, R. H.; Anastasiadis, S. H.; Fytas, G. J. Chem. Phys. 1996, 105, 3777.

(10) Kamath, S.; Colby, R. H.; Kumar, S. K. Macromolecules 2003, 36,8567 .

(11) Lodge, T.; McLeish, T. C. B. Macromolecules 2000, 33, 5278.

(12) Haley, J. C.; Lodge, T. P.; He, Y.; Ediger, M. D.; von Meerwall, E. D.; Mijovic, J. Macromolecules 2003, 36, 6142.

(13) Alegria, A.; Telleria, I.; Colmenero, J. J. Non-Cryst. Solids 1994, $172,961$.

(14) Roland, C. M.; Santangelo, P. G.; Baram, Z.; Runt, J. Macromolecules 1994, 27, 5382.

(15) Roland, C. M.; Santangelo, P. G.; Ngai, K. L.; Meier, G. Macromolecules 1993, 26, 6164 .

(16) Roland, C. M. Macromolecules 1995, 28, 3463

(17) Lutz, T. R.; He, Y.; Ediger, M. D.; Cao, H.; Lin, G.; Jones, A. A. Macromolecules 2003, 36, 1724

(18) Ngai, K. L.; Roland, C. M. Macromolecules 2004, 37, 2817.

(19) Ngai, K. L.; Roland, C. M. Rubber Chem. Technol. 2004, 77, 579 .

(20) Williams, G. In Dielectric Spectroscopy of Polymeric Materials Runt, J. P., Fitzgerald, J. J., Eds.; American Chemical Society: Washington, DC, 1997; Chapter 1.

(21) Naoki, M.; Katahira, S. J. Phys. Chem. 1991, 95, 431.

(22) Ferrer, M. L.; Lawrence, Ch.; Demirjian, B. G.; Kivelson, D.; Alba-Simionesco, C.; Tarjus, G. J. Chem. Phys. 1998, 109 , 8010.

(23) Paluch, M.; Casalini, R.; Roland, C. M. Phys. Rev. B 2002, $66,092202$.

(24) Roland, C. M.; Paluch, M.; Pakula, T.; Casalini, R. Philos. Mag. 2004, 84, 1573

(25) Roland, C. M.; Hensel-Bielowka, S.; Paluch, M.; Casalini, R. Rep. Prog. Phys. 2005, 68, 1405.

(26) Casalini, R.; Roland, C. M. Phys. Rev. E 2004, 69, 062501.

(27) Casalini, R.; Roland, C. M. Colloid Polym. Sci. 2004, 283, 107.

(28) Roland, C. M.; Capaccioli, S.; Lucchesi, M.; Casalini, R. J. Chem. Phys. 2004, 120, 10640.

(29) Van Krevelen, D. W. Properties of Polymers; Elsevier: New York, 1990

(30) Roland, C. M.; Paluch, M.; Casalini, R. J. Polym. Sci., Polym. Phys. 2004, 42, 4313 .

(31) Casalini, R.; Roland, C. M. Macromolecules 2005, 38, 1779.

(32) Ngai, K. L.; Casalini, R.; Roland, C. M. Macromolecules 2005, $38,4363$.

(33) Takeno, H.; Koizumi, S.; Hasegawa, H.; Hashimoto, T. Macromolecules 1996, 29, 2440.

(34) Zhang, S. H.; Jin, X.; Painter, P. C.; Runt, J. Polymer 2004, $45,3933$.

(35) Takahara, S.; Ishikawa, M.; Yamamuro, O.; Matsuo, T. J. Phys. Chem. B 1999, 103, 3288.

(36) Koplinger, J.; Kasper, G.; Hunklinger, S. J. Chem. Phys. 2000, $113,4701$.

(37) Floudas, G.; Fytas, G.; Reisinger, T.; Wegner, G. J. Chem. Phys. 1999, 111, 9129

(38) Alegría, A.; Gómez, D.; Colmenero, J. Macromolecules 2002 352030.
(39) Floudas, G. In Broadband Dielectric Spectroscopy; Kremer, F., Schonhals, A., Eds.; Springer-Verlag: Berlin, 2003; Chapter 8.

(40) Mpoukouvalas, K.; Floudas, G.; Zhang, S. H.; Runt, J. Macromolecules 2005, 38, 552 .

(41) Zhang, S. H.; Casalini, R.; Runt, J.; Roland, C. M. Macromolecules 2003, 36, 9917.

(42) Messe, L.; Prud'homme, R. E. J. Polym. Sci., Polym. Phys. Ed. 2000, 38, 1405 .

(43) Uchiyama, A.; Yatabe, T. Jpn. J. Appl. Phys. 2003, 42, 5665.

(44) Dikshit, A. K.; Kaito, A. J. Appl. Polym. Sci. 2004, 91, 2789.

(45) Cai, H.; Ait-Kadi, A.; Brisson, J. Polymer 2003, 44, 1481.

(46) Chin, Y. H.; Zhang, C.; Wang, P.; Inglefield, P. T.; Jones, A. A.; Kambour, R. P.; Bendler, J. T.; White, D. M. Macromolecules 1993, 26, 5372 .

(47) Chin, Y. H.; Inglefield, P. T.; Jones, A. A. Macromolecules 1993, 26,5372

(48) Zhao, J.; Chin, Y. H.; Liu, Y.; Jones, A. A.; Inglefield, P. T.; Kambour, R. P.; White, D. M. Macromolecules 1995, 28, 3881

(49) Robertson, C. G.; Wilkes, G. L. J. Polym. Sci., Polym. Phys. Ed. 2001, 39, 2118.

(50) Zoller, P.; Hoehn, H. H. J. Polym. Sci., Polym. Phys. Ed. 1982, $20,1385$.

(51) Zoller, P.; Walsh, D. J. Standard Pressure-Volume-Temperature Data for Polymers; Technomic: Lancaster, PA, 1995

(52) Roland, C. M.; Casalini, R. J. Chem. Phys. 2003, 119, 1838.

(53) Fox, T. G. Bull. Am. Phys. Soc. 1956, 1, 123.

(54) Leroy, E.; Alegria, A.; Colmenero, J. Macromolecules 2003, 36,7280

(55) An, L.; He, D.; Jing, J.; Wang, Z.; Yu, D.; Jiang, B.; Jiang, Z.; Ma, R. Eur. Polym. J. 1997, 33, 1523.

(56) Casalini, R.; Roland, C. M. J. Chem. Phys. 2003, 119, 4052

(57) Roland, C. M. Rubber Chem. Technol. 1989, 62, 456.

(58) Patterson, D. Polym. Eng. Sci. 1982, 22, 64.

(59) Cendoya, I.; Alegria, A.; Alberdi, J. M.; Colmenero, J.; Grimm, H.; Richter, D.; Frick, B. Macromolecules 1999, 32, 4065.

(60) Urakawa, O.; Sugihara, T.; Adachi, K. Polym. Appl. (Jpn.) 2002, 51, 10 .

(61) Zetsche, A.; Kremer, F.; Jung, H.; Schultze, H. Polymer 1990, $31,1988$.

(62) Ougizawa, T.; Dee, G. T.; Walsh, D. J. Macromolecules 1991, 24,3834 .

(63) Zoller, P. In Polymer Handbook, 3rd ed.; Brandrup, J., Immergut, H., Eds.; Wiley: New York, 1989.

(64) Williams, G. Trans. Faraday Soc. 1965, 61, 1564.

(65) Ngai, K. L.; Roland, C. M.; O’Reilly, J. M.; Sedita, J. S. Macromolecules 1992, 25, 3906.

(66) Ngai, K. L. J. Phys. Chem. B 1999, 103, 10684.

(67) Garrahan, J. P.; Chandler, D. Phys. Rev. Lett. 2002, 89, 035704.

(68) Garrahan, J. P.; Chandler, D. Proc. Natl. Acad. Sci. U.S.A. 2003, 100, 9710.

MA051481F 